\title{
LA ÉTICA EN LAS INTERVENCIONES SOCIALES: ALGUNOS MODELOS DE RESOLUCIÓN DE DILEMAS ÉTICOS
}

\section{THE ETHICS IN SOCIAL INTERVENTIONS: SOME MODELS OF DILEMMAS RESOLUTIONS ETHICS}

\author{
María Jesús Úriz Pemán y Francisco Idareta Goldaracena \\ Profesor/a Titular de Trabajo Social de la Universidad Pública de Navarra
}

\begin{abstract}
Resumen: A lo largo del presente artículo nos proponemos un doble objetivo: por un lado, destacar la importancia de la mirada ética como un elemento fundamental en las intervenciones sociales. Por otro, plantear la necesidad de contar con herramientas concretas para la toma de decisiones éticas. Para ello, explicaremos brevemente dos modelos de toma de decisiones: el de Loewenberg y Dolgoff (basado en una jerarquía entre principios éticos) y la propuesta no jerarquizada de Patricia Kenyon.
\end{abstract}

Palabras-clave: Ética, Intervención social, Dilemas éticos, Toma de decisiones éticas, Modelos de resolución de dilemas éticos, Jerarquías entre principios éticos.

Abstract: The aims of this article are to emphasize the importance of the ethical view as a fundamental element in social interventions, and to analyze tools for making ethical decisions. We will briefly explain two decision-making models: Loewenberg and Dolgoff (based on a hierarchy of ethical principles) and Patricia Kenyon's non-hierarchical proposal.

Key words: Ethics, Social intervention, Ethical dilemmas, Ethical decision-making, Models of ethical dilemma resolution, Hierarchies among ethical principles.

\section{Introducción}

La ética debe ser uno de los pilares fundamentales de todas las intervenciones profesionales, tanto las que se realizan en el ámbito de lo social como también las que suceden en otras áreas como la sanitaria o las profesiones libera- 
les. La mirada de las y los profesionales siempre debe estar enraizada en los principios éticos, en la deontología profesional y en los valores y virtudes necesarias para el cuidado y la atención de las personas usuarias. Sólo de este modo garantizaremos que las intervenciones profesionales sean, no sólo técnicamente buenas, sino que también respeten los principios éticos y los valores indispensables en las interacciones con las personas usuarias (Idareta, 2013; Idareta, Úriz, Viscarret, 2017).

En línea directa con este planteamiento, durante los últimos años en el ámbito académico estamos asistiendo a una creciente preocupación por las éticas profesionales, lo cual se ha reflejado directamente en un incremento en el número de publicaciones en torno a este campo. El caso del Trabajo Social también es un buen reflejo de este hecho, lo cual es especialmente significativo si miramos las recientes publicaciones de habla inglesa de la última década: Beckett \& Maynard, 2005; Bowles, Collingridge, Curry \& Valentine, 2006; Reamer, 2006; Banks \& Gallagher, 2009; Congress, Black \& Strom-Gottfried, 2009; Dolgoff, Loewenberg \& Harrington, 2009.

Pues bien, la mirada de la ética en las intervenciones sociales también hace que nos demos cuenta de la existencia de multitud de conflictos éticos. La mirada ética nos hace reflexionar cuando se nos presenta un conflicto entre principios éticos, cuando no sabemos muy bien cómo aplicar algunos valores morales, cuando nos encontramos con situaciones en las que ninguna opción moral no satisface plenamente... En definitiva, gracias a la mirada ética somos capaces, en primer lugar, de detectar los dilemas éticos y, en segundo lugar, de iniciar un proceso reflexivo y deliberativo encaminado a encontrar posibles soluciones a esos dilemas.

Hay que tener en cuenta, además, que los dilemas éticos pueden darse en muchos niveles: en la intervención directa con las personas usuarias, en situaciones en las que nuestros propios valores profesionales chocan con los de la institución en la que trabajamos, en la interrelación con profesionales de otras instituciones...

Cuando utilizamos la expresión "dilemas éticos" lo hacemos, no sólo para referirnos a situaciones en las que existe un conflicto entre principios éticos (entre autonomía y bienestar, por ejemplo), sino también a aquellas situaciones en las que encontramos argumentos morales, tanto para realizar una acción como 
para no realizarla (Bowles et al, 2006). Se trata, por tanto, de situaciones en las que los argumentos morales que encontramos no determinan un único curso de acción y precisamente por ello representan para nosotros un dilema ético, ya que ninguno de los caminos que podemos tomar nos convence plenamente (Banks \& Williams, 2005).

Pero, ¿cuáles son los principales dilemas éticos que han de resolver las trabajadoras y trabajadores sociales en España? A partir de los resultados que obtuvimos mediante el proyecto de investigación nacional de $\mathrm{I}+\mathrm{D}+\mathrm{i}$ sobre " $\mathrm{Di}$ lemas éticos de la intervención social: la perspectiva de los trabajadores sociales en España" realizado por el grupo EFIMEC ${ }^{1}$ entre los años 2009 y 2011 (Ballestero, Úriz y Viscarret, 2013), concluimos que los dilemas más frecuentes eran los relacionados con: el deber o no de informar a terceras personas (por ejemplo, cuando esas terceras personas demandaban información acerca de las y los usuarios); otras circunstancias en las que no estaba muy claro hasta dónde había que respetar o no la autonomía de las personas usuarias (por ejemplo, porque respetando su autonomía se ponía en peligro su bienestar); también situaciones relacionadas con el tratamiento de la información confidencial (desde cómo guardar la información confidencial hasta en qué situaciones estaría justificado romper dicha confidencialidad).

Además de esos tipos de dilemas éticos, aparecían también otros relacionados con: la duración de la intervención (cuando, por ejemplo, las profesionales se veían en la necesidad de finalizar la intervención, pero sin que hubieran cubierto plenamente las demandas y necesidades de las personas usuarias); dilemas en torno a la justa distribución de los recursos disponibles; otras situaciones en las que las y los profesionales se planteaban si decir o no toda la verdad o mentir...

Es evidente, entonces, que en las intervenciones sociales surgen muchos dilemas éticos de muy distintos tipos. Pero, ¿cómo se toman las decisiones éticas cuando hay que resolver dichos dilemas? Tratando de dar respuesta a esta problemática y, como continuación al Proyecto antes citado, en el grupo EFIMEC elaboramos otro Proyecto Nacional sobre la "Toma de decisiones éticas en la

\footnotetext{
${ }^{1}$ Grupo de investigación “Ética, Filosofía y Metodología de las Ciencias” del Departamento de Trabajo Social de la Universidad Pública de Navarra.
} 
práctica profesional del Trabajo Social”. El objetivo era averiguar precisamente qué hacen los y las profesionales delTrabajo Social cuando se encuentran con un dilema ético, es decir, cómo resuelven dichos dilemas.

Una de las principales conclusiones a las que llegamos después de realizar este Proyecto fue precisamente la falta de herramientas concretas para la toma de decisiones éticas. Esto significa que, pese a que más del 90 por ciento de las y los trabajadores sociales en España reconocía haber tenido que enfrentarse a algún dilema ético, el 97 por ciento reconocían no conocer ningún modelo ni metodología para la toma de decisiones éticas. Cuando tenían algún dilema ético consultaban a un superior, lo discutían con un colega o, simplemente, recurrían al sentido común o la intuición. Resulta evidente, por tanto, la necesidad de profundizar en los procesos de toma de decisiones éticas para tratar de proporcionar a dichos profesionales algunas herramientas para la toma de decisiones éticas (Ballestero et al., 2014).

\section{Herramientas para resolver dilemas éticos}

Si revisamos la literatura de habla inglesa veremos que en los últimos años se está produciendo un aumento de publicaciones en torno a este tema y, afortunadamente, hay algunas propuestas metodológicas interesantes aplicables al ámbito de la intervención social (Harrington \& Dolgoff, 2008; Kenyon, 1998, Reamer, 2006; Úriz, 2004).

Una posible forma de clasificar esas propuestas es distinguir dos grupos: las que establecen algún tipo de jerarquías entre principios éticos y aquellas en las que los principios éticos no son tan relevantes o, al menos, no se propone una jerarquía concreta entre ellos. En el primer grupo podríamos situar propuestas tan interesantes como el procedimiento deliberativo procedente de la Bioética (Gracia, 1991) o el modelo agregado de Loewenberg y Dolgoff (1982). Es de destacar que, pese a la enorme dificultad de tratar de establecer una jerarquía universal entre principios éticos, ambos realizan propuestas jerárquicas concretas. En el caso del modelo deliberativo, tomando cuatro principios básicos (autonomía, bienestar, no-maleficencia y justicia) y, en la propuesta de Loewenberg y Dolgoff, jerarquizando siete principios éticos que veremos a continuación. 
Dentro del segundo grupo encontramos propuestas tan interesantes como la de Reamer (2006) pues, aunque es cierto que establece varias líneas de acción que implican priorizar los "bienes básicos", no puede ser considerado estrictamente como un modelo jerarquizado. También dentro de este segundo grupo podemos situar el modelo de Patricia Kenyon (1998).

Dado que, por motivos de extensión, ahora no podemos profundizar en todas y cada una de esas propuestas, hemos elegido dos de ellas (una de cada grupo): por un lado, el modelo agregado de Frank M. Loewenberg y Ralph Dolgoff (1982) y, por otro, el modelo de Patricia Keynon (1998).

Loewenberg y Dolgoff (1982) realizan su propuesta con la finalidad de evitar la improvisación y de racionalizar el proceso de toma de decisiones éticas en el ejercicio profesional del Trabajo Social. En principio, su aplicabilidad no se circunscribe estrictamente a cuestiones éticas, por lo que, como se deduce de la formulación de los once pasos iniciales, puede decirse que es un modelo genérico de toma de decisiones.

Este modelo está basado en teorías de diferentes autores y se caracteriza porque la toma de decisiones se realiza progresivamente: en un primer momento se proponen once pasos genéricos de aproximación a la casuística concreta; seguidamente, se utilizan dos instrumentos diferentes que orientan a las y los profesionales de la intervención social en la resolución ética del caso: primeramente se utiliza el Ethical Rules Screen (ERS) y, con posterioridad, el Ethical Principles Screen (EPS).

Los once pasos que proponen inicialmente son los siguientes: 1) identificar el problema y los factores que contribuyen a mantenerlo; 2) identificar las personas e instituciones relacionadas con dicho problema (clientes, otros profesionales, etc.); 3) identificar los valores relevantes a dicho problema que mantienen los diversos participantes identificados en el paso 2, así como los valores sociales, profesionales y personales del cliente y del trabajador; 4) identificar metas y objetivos cuya consecución puede resolver o reducir el problema; 5) identificar estrategias de intervención alternativas; 6) asegurar la efectividad y eficacia de cada alternativa en términos de las metas identificadas; 7) determinar quién debería estar implicado en la toma de decisiones; 8) seleccionar la estrategia más apropiada; 9) implantar la estrategia seleccionada; 10) hacer un segui- 
miento de la implantación, atendiendo especialmente a las consecuencias que no se habían anticipado previamente; y 11) evaluar los resultados e identificar problemas adicionales.

Una vez concluida esta primera aproximación, el proceso progresivo de toma de decisiones ética continúa con el ERS, compuesto por las siguientes tres normas éticas, que remiten a las y los profesionales a la utilización de su código deontológico de referencia: 1) comprobar si se pueden aplicar algunas reglas del código de ética, que estarían por encima de los valores personales; 2) si se pueden aplicar una o más reglas, hay que seguirlas; y 3) si no existen reglas aplicables o hay un conflicto entre ellas, utilizar el EPS.

Efectivamente, el ERS remite al siguiente instrumento, el EPS, cuando el código deontológico de referencia resulte muy difuso o poco concreto, así como cuando este no plantee ninguna directriz ética específica. Es decir, si las normas éticas que componen el ERS no permiten dirimir los problemas o dilemas éticos que se plantean, Loewenberg y Dolgoff proponen utilizar los principios éticos del EPS.

Tales principios éticos se encuentran jerárquicamente ordenados y son los siguientes: 1) protección de la vida humana (vida en sentido amplio, incluyendo también el bienestar del usuario y de otras personas); 2) igualdad y desigualdad: en principio, todas las personas merecen ser tratadas de forma igualitaria, pero pueden ser tratadas de forma diferente si esa desigualdad es relevante (por ejemplo, en casos de abusos de niños); 3) autonomía y libertad: el/la profesional del Trabajo Social respetará la autonomía y libertad del usuario siempre que ello no implique un daño importante para sí mismo o para otras personas (en este último caso, sería prioritario el primer principio). Además, hay que evaluar los riesgos y beneficios de cada acción; 4) principio del menor daño (elegir la opción que cause el menor daño posible); 5) principio de la calidad de vida (para los individuos y para la comunidad); 6) principio de privacidad y confidencialidad; y 7) principio de sinceridad.

Los principios éticos contenidos en el EPS serían por tanto los siguientes: autonomía y libertad (derecho del usuario a su autonomía e independencia), igualdad y desigualdad (todas las personas, en las mismas circunstancias, deben ser tratadas de la misma forma); hacer el menor daño posible (si el daño es inevitable, el trabajador o trabajadora social elegirá la opción que cause el menor 
daño posible); privacidad y confidencialidad (proteger, lo más ampliamente posible, la privacidad y confidencialidad de la información relativa a los usuario); protección de la vida (se debe proteger la vida lo más ampliamente posible; aunque no se define cuándo empieza la vida y cuándo acaba); calidad de vida (se deben elegir las opciones que promuevan la mejor calidad de vida posible); confianza y completa revelación (el trabajador o trabajadora social debería decir completamente la verdad y proporcionar a la persona usuaria toda la información disponible).

En síntesis, Loewenberg y Dolgoff proponen un modelo de resolución de toma de decisiones que combina una serie de pasos generales y unas normas éticas, con la jerarquización de siete principios éticos básicos: en un primer momento se realiza una aproximación genérica a las circunstancias éticas de cada caso, para posteriormente, apelar a las normas éticas del código deontológico de referencia y, caso de que no nos sean útiles, a los principios éticos básicos jerarquizados.

Es de destacar la valentía de esta propuesta, primero, al elegir siete principios éticos entre los muchos posibles implicados en las intervenciones sociales y, segundo, al tratar de establecer una jerarquía entre ellos que sea lo más universal posible y que se pueda aplicar, por tanto, a múltiples intervenciones sociales.

En cuanto al grupo de modelos que no establecen una jerarquía concreta entre principios éticos, queremos destacar el de Patricia Kenyon (1998), quien presenta una guía para la toma de decisiones éticas basada en el código deontológico de la National Organization for Human Service Education (NOHSE). Este modelo está elaborado teniendo en cuenta las perspectivas de diversas profesiones de la intervención social y propone diez pasos con una serie de indicaciones y preguntas:

1. Describir la cuestión o el dilema ético:

- ¿Quién está implicado? ¿Cuál es su implicación?

- ¿Qué tiene el dilema?

- ¿Qué implicaciones tiene? ¿Qué riesgos hay?

- ¿Cuáles son las características principales?

- ¿Qué tipo de asunto es? 
2. Considerar los aspectos éticos y jurídicos:

- Considera todas las directrices éticas y normas jurídicas.

- Identifica tus propios valores personales pertinentes para el asunto.

- Identifica los valores sociales o comunitarios pertinentes para el asunto.

- Identifica los estándares profesionales pertinentes.

- Identifica las leyes y regulaciones pertinentes.

- Aplica estas directrices.

3. Examinar todos los conflictos:

- Describe los conflictos que experimentas internamente.

- Describe los conflictos que experimentas y que son externos (implican a nosotros, usuarios, supervisor, profesional, etc.).

- Decide cuáles de estos conflictos son menos importantes (si el externo prima sobre el interno, si puedes minimizar alguno de ellos...).

4. Resolver los conflictos pidiendo ayuda, si la necesitas, para tomar la decisión:

- Consulta con otros colegas, expertos o supervisores.

- Consulta bibliografía profesional pertinente.

- Busca ayuda en organizaciones profesionales o comités de ética.

5. Generar todos los posibles cursos de acción.

6. Examinar y evaluar las alternativas de actuación (se intenta priorizar entre alternativas)

- Considera las preferencias de las personas usuarias y de las otras personas implicadas desde una comprensión total de sus valores y creencias éticas (p.e.: autonomía del usuario).

- Elimina las alternativas que sean incompatibles con los valores y creen- 
cias de la persona usuaria y de otras personas significativas implicadas en el caso (no intentes imponer tus propios valores).

- Elimina las alternativas que sean incompatibles con otras normativas relevantes pertinentes en el caso.

- Elimina las alternativas para las que no tienes apoyo ni recursos (sé realista).

- Elimina las alternativas que no se ajusten a los principios éticos de universalidad, publicidad y justicia.

- Con el propósito de auxiliar en este paso, P. Kenyon recoge las siguientes preguntas sobre la univer salidad: ¡la acción es aplicable a todas las personas en situaciones similares (incluyéndote a ti mismo/a)? ¿Recomendarías actuar así a otros profesionales? ¿Lo aprobarías si un colega lo hiciera?

- En relación a la publicidad señala las siguientes: ¿la acción se basa en estándares éticos reconocidos por todas las personas implicadas? ¿Explicarías esa acción a tus colegas o en público? ¿Aceptarían ellos esa explicación?

-Y con respecto a la justicia: ¿la acción trata a las personas justamente? ¿Harías lo mismo con otros usuarios en situación similar? ¿Harías lo mismo si la persona usuaria fuera conocida o influyente?

- Prevé las posibles consecuencias de las alternativas aceptables que te quedan (a corto y largo plazo; ciertas, probables, improbables, etc.).

- Prioriza las alternativas aceptables que te quedan.

- Para ayudar en este paso, Kenyon (1998) recoge las preguntas que proponen Loewenberg y Dolgoff (1982): ¿Qué alternativas protegen más los derechos y bienestar de los usuarios y de otras personas? ¿Qué alternativas protegen más los derechos e intereses de la sociedad? ¿Qué puedes hacer para minimizar los conflictos entre proteger derechos y bienestar de los usuarios, la sociedad, otros, etc.? ¿Qué alternativa causa "el menor daño" posible? 
7. Seleccionar y evaluar la actuación elegida (el mejor curso de acción):

- Especialmente si no hemos elegido la acción que estaba situada en primer lugar, tenemos que evaluar nuestra decisión, preguntándonos también si estamos siendo influidos por algún factor que no hubiéramos reconocido o si debiéramos reconsiderar algo.

8. Planificar la actuación: Desarrolla un plan de actuación y llévalo a cabo.

9. Evaluar el resultado de la acción que has llevado a cabo:

- ¿Los resultados eran los esperados? ¿Aún piensas que ésa era la mejor decisión?

10. Examinar las consecuencias / implicaciones:

- ¿Qué has aprendido del proceso y sus resultados? ¿Qué implicaciones tiene para futuras tomas de decisiones éticas?

\section{Conclusiones}

Como se puede ver, el modelo propuesto por Patricia Kenyon es muy reflexivo. Hace que nos planteemos muchas preguntas en cada uno de los pasos y también hace que abramos muchos cursos de acción para luego poder ir eliminando entre ellos las alternativas menos aceptables. En cuanto a los principios éticos, sólo aparecen tres en el paso seis: universalidad, publicidad y justicia. Las preguntas relacionadas con dichos principios nos ayudan a eliminar alternativas en la toma de decisiones éticas.

Es importante la adquisición de cierta práctica en la toma de decisiones éticas, comenzando por situaciones más simples y, después, aumentando su complejidad. De hecho, Keynon propone la toma de decisiones paso a paso para ir interiorizando cada uno de ellos y adquiriendo cierta habilidad para poder aplicarlos a situaciones cada vez más complejas. Todo ello con el propósito de dotar de recursos y herramientas a los profesionales para que puedan resolver más fácilmente los conflictos a los que se tengan que enfrentar.

En definitiva, sea cual sea el modelo que elijamos para la toma de decisiones éticas, lo importante es que sea un proceso deliberado, argumentado; un 
proceso reflexivo en el que nos planteemos las posibles alternativas y las consecuencias de una u otra decisión. También es necesario tener en cuenta el marco legislativo, acudir a la deontología profesional y, si es posible, no tomar la decisión de forma aislada, sino dentro del equipo de trabajo o consultando a un comité de ética. Tomemos la decisión que tomemos, hemos de ser capaces de justificar razonadamente dicha decisión.

La ética no es una ciencia exacta, por lo que nunca sabremos a un cien por cien si la opción que hemos elegido ante un dilema ético es la mejor opción posible. Pero de lo que sí tenemos que asegurarnos es de haber realizado un proceso reflexivo, deliberativo, en el que hayamos tenido en cuenta los valores y principios éticos implicados; un proceso en el que abramos todos los posibles cursos de acción y en el que nos planteemos las consecuencias que se derivan de cada uno de ellos. Se trata, en definitiva, de que ningún elemento se quede fuera de la reflexión ética. De este modo, poco a poco conseguiremos el objetivo de interiorizar esa mirada ética tan necesaria en todas las intervenciones profesionales.

\section{Bibliografía}

BALLESTERO-IZQUIERDO, A., ÚRIZ-PEMÁN, M.J., y VISCARRET-GARRO, J.J. (2013) ¿Qué dilemas éticos encuentran los profesionales del Trabajo Social en España y cómo los resuelven?. Quins dilemes ètics troben els professionals del treball social a Espanya i com els resolen?, Revista de Treball Social, 199, 35-45.

BALLESTERO-IZQUIERDO, A., IDARETA-GOLDARACENA, F., ÚRIZ-PEMÁN, M.J., VISCARRET-GARRO, J.J., y DIEZ-LUQUI, G. (2014). Cómo afrontar los dilemas éticos en la práctica profesional del trabajo social. E. Pastor-Seller (Editor), Las prácticas curriculares en el Grado de Trabajo Social. Supervisión y construcción del conocimiento desde la práctica profesional (pp. 227 - 246). Madrid: Editorial Universitas.

BANKS, S., \& WILLIAMS, R. (2005) “Accounting for Ethical Difficulties in Social Welfare Work: Issues, Problems and Dilemmas”, British Journal of SocialWork, 35, 1005-1022.

BANKS, S., \& GALLAGHER, A. (2009). Ethics in professional life: virtues for health and social care. Basingstoke: Palgrave Macmillan.

BECKETT, C., \& MAYNARD, A. (2005). Values and ethics in social work:An introduction. London: Sage. BOWlES, W., COLLINGRIDGE, M., CURRY, S., \& VALENTINE, B. (2006). Ethical practice in social work: An applied approach. Crow's Nest, New South Wales: Allen and Unwin.

CONGRESS, E., BLACK, P., \& STROM-GOTTFRIED, K. (Eds.). (2009). Teaching social work ethics and values: A curriculum resource. Alexandra, Va: Council on Social Work Education. 
DOLGOFF, R., LOEWENBERG, F., \& HARRINGTON, D. (2009). Ethical decisions for social work practice. (8th ed.) Belmont, CA: Brooks Cole.

GRACIA, D. (1991) Procedimientos de decisión en ética clínica. Madrid: Eudema.

HARRINGTON, D., \& DOLGOFF, R. (2008) "Hierarchies of Ethical Principles for Ethical Decision Making in Social Work". Ethics and SocialWelfare, 2 (2), 183-196.

KEYNON, P. (1998) What would you do? An ethical case workbook for human service professsionals. USA: Brooks Cole Publishing Company.

LOEWENBERG, F.M., y DOLGOFF, R. (1982). Ethical Decissions for SocialWork Practice. Ithaca (Illinois): F.E. Peacock Pub.

REAMER, F. (2006). Social work values and ethics. (3rd ed.). New York: Columbia University Press.

ÚRIZ M. J. (2004) “Modelos de resolución de dilemas éticos en Trabajo Social”. RTS, Revista de Trabajo Social, 175, 6-27. 